\section{Compreensão de informações relativas ao tratamento anti-retroviral entre indivíduos infectados pelo HIV}

\author{
HIV patients' understanding of information \\ on antiretroviral therapy
}

Maria das Graças Braga Ceccato 1,2

Francisco A. Acurcio 1,2

Palmira de Fátima Bonolo 2

Gustavo M. Rocha 2

Mark D. C. Guimarães 2

\footnotetext{
1 Faculdade de Farmácia, Universidade Federal de Minas Gerais, Belo Horizonte, Brasil.

2 Faculdade de Medicina, Universidade Federal de Minas Gerais Belo Horizonte, Brasil.

Correspondência F. A. Acurcio Faculdade de Farmácia Universidade Federal de Minas Gerais. Av. Olegário Maciel 2360 Belo Horizonte, $M G$ 30180-112, Brasil. acurcio@medicina.ufmg.br
}

\begin{abstract}
To assess the understanding of information related to antiretroviral therapy among HIV-infected patients enrolled in public AIDS services (Belo Horizonte, Minas Gerais State, Brazil), a crosssectional analysis was carried out, based on interviews with patients after initial provision of antiretroviral drugs. The study evaluated the information on antiretroviral therapy provided by healthcare professionals and the patients' level of understanding in relation to prescription information. This level was classified as insufficient if there was disagreement of more than $30.0 \%$ between the information reported by the patient and the written prescription. Divergence between prescriptions and information reported by 358 interviewed patients was observed. The level of understanding regarding the prescribed antiretroviral was obtained, and $26.3 \%$ of patients displayed insufficient understanding. The results show an important proportion of patients with misunderstanding of information regarding antiretroviral therapy, mainly those with limited schooling and low income. It is necessary to bolster strategies to increase quality of recommendations provided to these patients. Improving the multidisciplinary team approach to patient care should help reverse the observed situation.
\end{abstract}

Acquired Immunodeficiency Syndrome; Highly Active Antiretroviral Therapy; Period Analisys

\section{Introdução}

A epidemia de HIV/AIDS configura um dos mais sérios problemas contemporâneos de saúde pública, apresentando alto grau de morbimortalidade e perspectivas de um contínuo crescimento e propagação em todos os continentes. As possibilidades de controle dessa pandemia ainda parecem remotas, apesar do desenvolvimento de novas terapias e do esforço mundial na busca de uma vacina eficaz contra a infecção. Dados da UNAIDS - Joint United Nations Programme on HIVIAIDS (http://www. unaids.org/en/Resources/Publications/Corporate+publications/AIDS+epidemic +up+date ++December+2003.asp, acessado em 04/Dez/ 2003), divulgados em dezembro de 2003, estimam 34 a 46 milhões de pessoas vivendo atualmente com o vírus, sendo aproximadamente metade do sexo feminino. É a quarta maior causa mundial de mortalidade; somente no ano de 2002 morreram entre 2,50-3,51 milhões de pessoas (http://www.unaids.org/en/Resources/ Publications / Corporate+publications / AIDS+epidemic+up+date+-+December+ 2003.asp, acessado em 04/Dez/2003). A América Latina é a região que ocupa a quarta posição em número de infectados no mundo, sendo o Brasil o país que possui o maior número de casos de AIDS na região (http://www.unaids. org/en/Resources/Publications/Corporate+ publications/AIDS+epidemic+up+date+-+De- 
cember+2003.asp, acessado em 04/Dez/2003), com 277.141 casos registrados até setembro de 2003, distribuídos de forma heterogênea em todas as Unidades da Federação, sendo que desses, 70,0\% eram do sexo masculino (http:www. aids.gov.br/imprensa/NoticiasImpressao.asp? NOTCod=52007, acessado em 04/Dez/2003). A evolução do conhecimento e os avanços na utilização de recursos diagnósticos e terapêuticos, principalmente com o advento da terapia combinada de alta potência (HAART) em 1996, têm se mostrado decisivos no aumento da sobrevida de indivíduos com infecção pelo HIV. A eficácia das associações terapêuticas aliada à acessibilidade aos medicamentos permitem, atualmente, classificar essa infecção como uma doença crônica, deslocando progressivamente a atenção aos pacientes para a rede ambulatorial. No Brasil, dentre as estratégias para combater a epidemia destaca-se a política de distribuição dos medicamentos anti-retrovirais aos portadores do HIV que necessitem de tratamento. Vários indicadores evidenciam o efeito positivo dessa política adotada no país, como uma redução da mortalidade $(50,0 \%)$, diminuição das internações hospitalares $(80,0 \%)$, redução da incidência de infecções oportunistas, e da transmissão vertical, dentre outros 1. A efetividade da terapia HAART é dependente de uma estreita adesão ao regime prescrito, pois o uso irregular ou em doses insuficientes pode propiciar o desenvolvimento de vírus HIV resistente. Conseqüências de uma baixa adesão incluem limitações terapêuticas para o paciente e ameaça para a saúde pública, diante da possibilidade de transmissão de vírus multirresistentes 2,3 . Na terapia combinada é necessário atingir alta proporção de uso dos medicamentos indicados (90,0\%-100,0\%) para suprimir a replicação viral 4 . Os atuais esquemas terapêuticos são complexos, de difícil adesão e associados a reações adversas e interações medicamentosas. A não-adesão é a causa mais comum da falha do tratamento e é a principal variável na qual os serviços de saúde podem intervir para aumentar a efetividade da medicação. Dentre vários fatores que podem levar o indivíduo a não aderir ao tratamento destacamos a compreensão insuficiente sobre o uso dos medicamentos, bem como a falta de informação sobre os riscos advindos da não-adesão 5. Uma vez que as pessoas não podem tomar os medicamentos corretamente se não sabem como fazê-lo, fornecer informações sobre os medicamentos constitui atividade fundamental para promover a adesão. Entretanto, essa prática não tem sido satisfatória no processo de atendimento ao paciente. Pesquisadores observaram que $17,0 \%$ a 30,0\% dos pacientes com novas prescrições não receberam informações verbais de seus médicos e $30,0 \%$ a $87,0 \%$ não as receberam dos farmacêuticos 6 . Assinalaram, ainda, a ausência de informações acerca dos medicamentos como uma das principais razões pelas quais $30,0 \%$ a $50,0 \%$ dos pacientes não os usaram conforme a prescrição 7. Uma comunicação inadequada entre o paciente e o profissional da saúde sobre os medicamentos e o cumprimento da prescrição, tem sido apontada como um dos principais fatores responsáveis pelo uso em desacordo com a prescrição médica 8,9,10 e, constitui um dos mais freqüentes relatos de dificuldades na relação médicopaciente, durante entrevistas com indivíduos infectados pelo HIV ${ }^{11}$. A literatura enfatiza a importância de fornecer aos pacientes informações por escrito, como meio de reforçar as instruções verbais e assim aumentar a compreensão do regime terapêutico prescrito 12,13,14 . No entanto, aspectos específicos relativos à informação obtida pelo paciente sobre os medicamentos prescritos têm sido pouco estudados no Brasil 15. Os trabalhos mencionados acima permitem vislumbrar a importância da adequada comunicação entre os profissionais de saúde e o paciente no processo de atenção à saúde. Nesse sentido, o objetivo principal deste estudo é avaliar a compreensão de informações relativas ao tratamento anti-retroviral entre indivíduos infectados pelo HIV, na perspectiva de contribuir para a melhoria da adesão a esse tratamento, reduzindo assim a possibilidade de desenvolvimento de cepas multirresistentes em razão de um uso inadequado dos medicamentos

\section{Material e métodos}

Este trabalho integra o Projeto Adesão ao Tratamento Anti-retroviral - Projeto ATAR (Guimarães MDC et al. Fatores Associados à Adesão ao Tratamento Anti-retroviral (ARV) em Indivíduos Infectados pelo HIVIAIDS: Uma Abordagem Quantitativa e Qualitativa, Belo Horizonte (MG), 2001-2003 (Projeto ATAR). Relatório Técnico apresentado ao Programa Nacional de DST/AIDS; 2003) um estudo prospectivo da adesão ao tratamento anti-retroviral em indivíduos infectados pelo HIV/AIDS, em Belo Hori- 
zonte, Minas Gerais, Brasil, no período de 2001 a 2002, desenvolvido pelo Grupo de Pesquisa em Epidemiologia e Avaliação em Saúde da Universidade Federal de Minas Gerais (UFMG). Foi realizada uma análise transversal de aspectos relacionados à compreensão de informações sobre os medicamentos prescritos, obtidos na entrevista basal do estudo prospectivo. Indivíduos infectados pelo HIV, inscritos em serviços públicos de saúde e admitidos para a primeira prescrição de anti-retrovirais, foram entrevistados logo após a primeira dispensação desses medicamentos. O estudo foi realizado em dois serviços públicos de saúde de referência para a assistência ambulatorial especializada em HIV/AIDS, em Belo Horizonte: Centro de Treinamento e Referência em Doenças Infecto-Parasitárias Orestes Diniz, vinculado à Secretaria Municipal de Saúde de Belo Horizonte em convênio com a UFMG; e Ambulatório do Hospital Eduardo de Menezes, vinculado à Fundação Hospitalar de Minas Gerais. As entrevistas tiveram início em maio de 2001 e término em maio de 2002. O público-alvo e os critérios de elegibilidade adotados pelo Projeto ATAR incluíram todos os indivíduos com evidência laboratorial de infecção pelo HIV e/ou diagnóstico de AIDS, com idade igual ou superior a 18 anos, inscritos no Centro de Treinamento e Referência em Doenças Infecto-Parasitárias Orestes Diniz ou Hospital Eduardo de Menezes, admitidos para sua primeira prescrição, com esquema duplo, tríplice ou com mais combinações da terapia anti-retroviral e que consentiram em participar do estudo. Foram excluídos os indivíduos que não apresentaram condições mínimas de autonomia para decidir acerca da adesão ao tratamento e os indivíduos em uso de medicamentos devido a acidente de trabalho. O projeto foi avaliado e aprovado pelas instituições onde a pesquisa foi desenvolvida e pelo Comitê de Ética em Pesquisa da UFMG. O princípio de confidencialidade foi rigorosamente cumprido, incluindo a apresentação dos dados de forma agregada e sem a identificação dos participantes na descrição dos resultados. A coleta dos dados foi obtida por meio de instrumento previamente testado e após a capacitação dos entrevistadores. Foram investigadas características sócio-demográficas, características relacionadas com a terapia anti-retroviral e com a orientação proporcionada pelos profissionais de saúde. Verificou-se a ocorrência de orientação realizada pelo médico e pelo farmacêutico quanto a aspectos importantes relacionados à terapia anti-retroviral, como nome, horário, dose, alimentação, conduta ao esquecer de tomar, uso con- comitante de álcool, efeitos colaterais/reações adversas, conseqüências da interrupção do tratamento, data de retorno para buscar medicamentos. Também foi investigado o nível de entendimento desta orientação (completo, elevado, mediano, baixo ou nenhum) segundo a percepção do paciente e baseada nas respostas à pergunta “Em relação à (s) orientação (ões) que recebeu do seu médico (farmacêutico), como você entendeu o que foi dito?".

O nível de compreensão da prescrição de anti-retrovirais foi calculado adotando-se os seguintes procedimentos: inicialmente a receita médica foi transcrita, em forma de respostas às perguntas realizadas durante as entrevistas; a seguir, o nível de compreensão da prescrição de cada medicamento foi medido por meio de um escore, atribuindo-se diferentes pontos para cada item, de acordo com sua importância para o uso seguro dos medicamentos, em que houve concordância entre a resposta do paciente e a informação na prescrição. O uso seguro de medicamentos foi aqui entendido como aquele que não cause danos à saúde e ao bemestar do paciente. Para as informações relativas às reações adversas, precauções e recomendações quanto à alimentação, considerouse a possível ocorrência de orientação verbal e, quando a informação não constava na receita, comparou-se a resposta do paciente com as informações existentes no Consenso Brasileiro 5 . O nível global de compreensão da prescrição pelo paciente foi, então, calculado pela média dos escores obtidos para cada um dos anti-retrovirais prescritos. Esse procedimento baseou-se na proposição de Silva et al. 15 , modificada, atribuindo-se a seguinte pontuação: (a) nome do medicamento, dose e freqüência $=2$ pontos para cada item; (b) reações adversas, indicação e duração do tratamento = 1 ponto; e (c) precauções e alimentação $=0,5$ ponto. Para o item indicação, avaliou-se o conhecimento do paciente sobre o modo de ação dos anti-retrovirais. Obteve-se, assim, um máximo de 10,0 pontos. O nível de compreensão da prescrição de anti-retrovirais foi classificado como suficiente quando o escore foi igual ou superior a 7,0 pontos (isto é $70,0 \%$ ), e estabelece condições de uso do medicamento de forma segura; e insuficiente quando o escore foi menor que 7,0 pontos. As modificações na proposição original de Silva et al. 15 consistiram na inclusão do item alimentação para a atribuição de pontos, além da elevação do ponto de corte para a classificação do nível insuficiente de compreensão, de 6,0 para 7,0 pontos. Optou-se assim, neste trabalho, por uma definição um pouco mais rigorosa quanto às condições de uso do medica- 
mento de forma segura. No momento da entrevista, permitiu-se ao paciente consultar a receita ou embalagem do medicamento, ou qualquer anotação que ele tivesse em mãos. Quando o paciente não possuía a receita em mãos, os dados foram buscados na segunda via da receita arquivada na farmácia. Nos casos em que não foi possível a recuperação da receita, o paciente foi excluído do estudo.

As informações obtidas foram organizadas em um banco de dados, utilizando-se o programa Paradox ${ }^{\circledR}$ versão 4.5 , e para viabilizar as análises estatísticas utilizou-se SAS ${ }^{\circledR}$ versão 6.11 e o Epi Info 2002. Realizou-se uma análise descritiva dos dados, que incluiu descrição da população estudada, distribuições de freqüência e medidas estatísticas de resumo das variáveis selecionadas. Procedeu-se a análise de concordância entre as respostas dos entrevistados e as informações contidas nas receitas médicas. Calculou-se, então, o nível de compreensão da prescrição de cada medicamento anti-retroviral e o nível global de compreensão do tratamento.

\section{Resultados}

Dos 406 participantes recrutados para o Projeto ATAR, 48 (12,0\%) foram excluídos desta análise devido à indisponibilidade da receita médica, resultando em uma amostra final de 358 pacientes. As características sociodemográficas apresentadas na Tabela 1 , mostram predominância do sexo masculino (55,6\%), a maioria da população tinha trinta anos ou mais $(67,9 \%$; mediana $=33,0$ anos; média $\pm \mathrm{DP}=34,7 \pm 9,7$ anos), viviam sós $(60,1 \%)$, com menos de oito anos de estudo (51,5\%; mediana $=7,0$ anos; média $\pm \mathrm{DP}=7,3 \pm 3,5$ anos) e com renda inferior a $\mathrm{R} \$ 350,00$ (70,5\%; mediana $=\mathrm{R} \$ 160,00$; média $\pm \mathrm{DP}=\mathrm{R} \$ 347,00 \pm \mathrm{R} \$ 599,50)$.

Da população investigada, apenas $8,1 \%$ usaram esquema mono ou duplo e 91,9\% usaram esquema tríplice ou com mais combinações de anti-retrovirais. Dos pacientes com esquema tríplice ou com mais combinações, 52,5\% fizeram uso de inibidor de protease. Dentre os 767 anti-retrovirais prescritos, a Zidovudina associada à Lamivudina (AZT + 3TC) apresentou-se como o anti-retroviral mais prescrito $(35,2 \%)$, seguido pelo Nelfinavir (16,6\%) e Efavirenz (12,3\%). A utilização de outra medicação de uso contínuo ou regular diferente dos anti-retrovirais ocorreu para 145 (40,5\%) participantes. Quanto ao conhecimento sobre o modo de ação dos anti-retrovirais, investigado por meio de perguntas feitas durante a entrevista basal,

\begin{tabular}{|c|c|c|}
\hline \multicolumn{3}{|c|}{$\begin{array}{l}\text { Distribuição de freqüência da população } \\
\text { estudada de acordo com as características } \\
\text { sócio-demográficas ( } n=358 \text { ). Belo Horizonte, } \\
\text { Minas Gerais, Brasil, } 2001 / 2002 \text {. }\end{array}$} \\
\hline Características & $\mathbf{n}$ & $\%$ \\
\hline \multicolumn{3}{|l|}{ Idade (em anos) } \\
\hline$<30$ & 115 & 32,1 \\
\hline$\geq 30$ & 243 & 67,9 \\
\hline \multicolumn{3}{|l|}{ Sexo } \\
\hline Masculino & 199 & 55,6 \\
\hline Feminino & 159 & 44,4 \\
\hline \multicolumn{3}{|l|}{ Estado civil } \\
\hline Solteiro/divorciado/separado & 215 & 60,1 \\
\hline Casado/união & 143 & 39,9 \\
\hline \multicolumn{3}{|l|}{ Escolaridade (em anos) } \\
\hline$<8$ & 184 & 51,5 \\
\hline$\geq 8$ & 173 & 48,5 \\
\hline \multicolumn{3}{|l|}{ Renda mensal } \\
\hline$<\mathrm{R} \$ 350,00$ & 249 & 70,5 \\
\hline$\geq R \$ 350,00$ & 104 & 29,5 \\
\hline
\end{tabular}

verificou-se que apenas $39,7 \%$ dos entrevistados disseram que o vírus não é destruído pelos anti-retrovirais, mas $92,2 \%$ afirmaram que esse tipo de medicamento não deixa o HIV aumentar. Uma proporção de $79,1 \%$ considerou que a terapia anti-retroviral melhora a resistência do organismo e para $84,1 \%$ ela ajuda a evitar algumas doenças graves. Além disso, 84,9\% têm consciência de que os anti-retrovirais devem ser tomados por toda a vida. Em síntese, o índice médio de acerto para as questões sobre o modo de ação dos anti-retrovirais foi de $74,9 \%$, o de erro $16,5 \%$ e cerca de $10,7 \%$ dos entrevistados não sabiam a resposta.

\section{Orientação dos profissionais de saúde segundo a percepção do paciente}

No que se refere ao número de profissionais de saúde provedores de orientação, $4,7 \%$ dos pacientes receberam orientações de três profissionais de saúde; $67,9 \%$, de dois; $20,1 \%$ de um e $7,3 \%$ dos pacientes ficaram sem nenhuma orientação profissional. Duzentos e oitenta e três pacientes $(79,4 \%)$ receberam orientação do médico, 297 (82,9\%) do farmacêutico e apenas $27(7,6 \%)$ receberam orientação do enfermeiro. É apresentada na Tabela 2 a distribuição de fre- 
qüência da ocorrência de orientação, realizada pelo médico e pelo farmacêutico, quanto a aspectos importantes da terapia anti-retroviral. Conforme se observa, tanto para o médico quanto para o farmacêutico, uma pequena proporção dos pacientes reportou ter sido orientada quanto à conduta ao esquecer de tomar o medicamento e ao uso concomitante de álcool, sendo que aproximadamente um terço não foi alertado quanto às conseqüências da interrupção do tratamento. As maiores proporções de orientação foram observadas para nome, horário e quantidade dos medicamentos. Em geral, as proporções de respostas afirmativas para orientação farmacêutica foram superiores àquelas para orientação médica.

O nível de entendimento da orientação médica ( $n=339$ ) segundo a percepção do paciente foi considerado completo, elevado, mediano ou baixo/nenhum por respectivamente, $55,8 \%$, $19,2 \%, 16,5 \%$ e 8,5\% dos entrevistados. Já para o nível de entendimento da orientação farmacêutica $(n=324)$ esses percentuais foram, respectivamente, $69,8 \%, 17,9 \%, 8,3 \%$ e $4,0 \%$. Observamos que houve uma maior proporção de pacientes que entenderam completamente a orientação farmacêutica, quando comparamos com os resultados da orientação médica.

\section{Nível de compreensão da prescrição de anti-retrovirais pelos pacientes}

Na Tabela 3 está sumarizada a concordância entre a informação relativa aos anti-retrovirais prescritos e a resposta do paciente em relação a cada item. Analisando-se o total de medicamentos anti-retrovirais prescritos, observou-se um índice de concordância de $92,8 \%$ para a dose a ser tomada, de $91,7 \%$ para freqüência de administração e de $72,5 \%$ para o nome do medicamento. Verificou-se uma proporção muito baixa de registro, nas receitas médicas, de informações relativas a reações adversas $(<1,0 \%)$, recomendações quanto à alimentação $(13,3 \%)$ e precauções a serem adotadas $(9,0 \%)$. Considerando-se a possibilidade de orientação verbal e comparando-se a resposta do paciente com a prescrição ou o Consenso Brasileiro 5, as proporções de concordância para estes três itens se elevam para $61,1 \%, 47,7 \%$ e $15,3 \%$, respectivamente. O índice de concordância para duração do tratamento foi de $84,9 \%$ e para indicação terapêutica de $71,2 \%$.

Na Tabela 4 está apresentada a distribuição de freqüência dos esquemas anti-retrovirais prescritos e os respectivos níveis de compreensão do tratamento pelos pacientes. Os esquemas anti-retrovirais incluíam inibidores da transcriptase reversa análogos de nucleosídeo (ITRN) e não análogos de nucleosídeo (ITRNN), além dos inibidores da protease (IP). O esquema mais prescrito foi o AZT + 3TC associado ao Nelfinavir [2 ITRN + 1 IP] $(26,0 \%)$, seguido pelo AZT + $3 \mathrm{TC}$ associado ao Efavirenz [2 ITRN + 1 ITRNN] $(22,9 \%)$ e AZT + 3TC associado ao Nevirapina [2 ITRN + 1 ITRNN] $(15,4 \%)$. Considerando a mediana, os melhores níveis de compreensão foram observados para os esquemas AZT + ddI + Nelfinavir e AZT + ddI + Indinavir. O nível global de compreensão da prescrição de anti-retrovirais apresentou média de $7,7(\mathrm{DP}=1,5$; Coeficiente de variação - CV $=19,3 \%$ ) e mediana de 8,1 , tendo sido classificado como insuficiente $(<7,0)$ para $94(26,3 \%)$ entrevistados.

Orientações realizadas pelo médico e pelo farmacêutico relativas aos medicamentos anti-retrovirais, na percepção dos pacientes ( $n=358$ ). Belo Horizonte, Minas Gerais, Brasil, 2001/2002.

\begin{tabular}{|c|c|c|c|c|}
\hline \multirow[t]{2}{*}{ Características } & \multicolumn{2}{|c|}{ Médico } & \multicolumn{2}{|c|}{ Farmacêutico } \\
\hline & $n^{*}$ & $\%$ & $n^{*}$ & $\%$ \\
\hline Conduta ao esquecer de tomar o anti-retroviral & 115 & 32,1 & 156 & 43,6 \\
\hline Uso concomitante de álcool & 154 & 43,0 & 169 & 47,2 \\
\hline Conseqüências ao parar de tomar o anti-retroviral & 225 & 62,8 & 231 & 65,5 \\
\hline Alimentação & 260 & 72,6 & 280 & 78,2 \\
\hline Efeitos colaterais/reações adversas & 266 & 74,3 & 272 & 76,0 \\
\hline Quando retornar para buscar anti-retroviral & 265 & 74,0 & 307 & 85,8 \\
\hline Nome & 303 & 84,6 & 310 & 86,6 \\
\hline Horários & 322 & 89,9 & 317 & 88,5 \\
\hline Quantidades & 323 & 90,2 & 316 & 88,3 \\
\hline
\end{tabular}

* respostas afirmativas para cada item. 
Concordância entre as informações relativas aos anti-retrovirais prescritos e as respostas dos pacientes. Belo Horizonte, Minas Gerais, Brasil, 2001/2002.

\begin{tabular}{lcc}
\hline Características & $\mathbf{n}$ & $\%$ \\
\hline Dose* $^{*}$ & 712 & 92,8 \\
Freqüência de administração* $^{*}$ & 704 & 91,7 \\
Nome do medicamento* $^{*}$ & 556 & 72,5 \\
Reações adversas* $^{*}$ & 469 & 61,1 \\
Alimentação* $^{*}$ & 366 & 47,7 \\
Precauções* $^{*}$ & 117 & 15,3 \\
Indicaçãão do & 304 & 84,9 \\
\hline
\end{tabular}

* o $\mathrm{n}$ se refere ao número de medicamentos prescritos $=767 ;{ }^{\star \star}$ o $\mathrm{n}$ se refere ao número de pacientes elegíveis participantes $=358$.

\section{Discussão}

As características sociodemográficas da população estudada mostram predominância de indivíduos do sexo masculino, com trinta anos ou mais, que viviam sós, com menos de oito anos de estudo e com renda inferior a $\mathrm{R} \$ 350,00$. Esse resultado é condizente com a literatura brasileira recente no que diz respeito a pacientes portadores de HIV/AIDS 3,11,16 e com dados do Ministério da Saúde 1. Quanto ao esquema terapêutico prescrito para a população investigada, mais de 90,0\% usaram esquema triplo ou com mais combinações de anti-retrovirais e desses, mais de 50,0\% fizeram uso de inibidor de protease. Embora este achado seja consensual com o de outros autores, a proporção de uso

Tabela 4

Distribuição de freqüência dos esquemas anti-retrovirais utilizados e respectivos níveis de compreensão do tratamento pelos pacientes. Belo Horizonte, Minas Gerais, Brasil, 2001/2002.

\begin{tabular}{|c|c|c|c|c|c|}
\hline \multirow[t]{2}{*}{ Esquema anti-retroviral } & \multicolumn{2}{|c|}{ Freqüência } & \multicolumn{3}{|c|}{ Nível de compreensão } \\
\hline & $\mathrm{n}$ & $\%$ & Média \pm DP & Mediana & $\mathrm{CV}$ \\
\hline$A Z T+d d l+N F V$ & 21 & 5,9 & $8,3 \pm 1,2$ & 8,6 & 13,9 \\
\hline$A Z T+d d l+I D V$ & 3 & 0,8 & $8,6 \pm 1,4$ & 8,5 & 15,7 \\
\hline$A Z T+3 T C+N F V$ & 93 & 26,0 & $7,8 \pm 1,6$ & 8,3 & 21,1 \\
\hline$A Z T+3 T C+N V P$ & 55 & 15,4 & $7,9 \pm 1,2$ & 8,3 & 15,8 \\
\hline $3 T C+d 4 T+E F Z$ & 3 & 0,8 & $8,1 \pm 0,9$ & 8,3 & 10,7 \\
\hline$d d l+d 4 T+N F V$ & 6 & 1,7 & $7,6 \pm 1,3$ & 8,2 & 17,3 \\
\hline$A Z T+3 T C+I D V$ & 19 & 5,3 & $7,3 \pm 1,6$ & 8,1 & 22,1 \\
\hline$d d l+d 4 T+E F Z$ & 5 & 1,4 & $7,8 \pm 1,1$ & 8,0 & 13,7 \\
\hline$A Z T+3 T C+E F Z$ & 82 & 22,9 & $7,7 \pm 1,3$ & 8,0 & 16,6 \\
\hline$A Z T+3 T C+I D V+R T V$ & 15 & 4,2 & $7,7 \pm 1,0$ & 8,0 & 12,4 \\
\hline $\mathrm{AZT}$ & 24 & 6,7 & $7,2 \pm 1,8$ & 7,7 & 24,9 \\
\hline$A Z T+d d l+N V P$ & 7 & 2,0 & $8,0 \pm 1,0$ & 7,5 & 12,3 \\
\hline $3 T C+d 4 T+N F V$ & 8 & 2,2 & $6,8 \pm 2,1$ & 7,2 & 30,1 \\
\hline $3 T C+d 4 T+N V P$ & 2 & 0,6 & $7,0 \pm 2,6$ & 7,0 & 37,0 \\
\hline$A Z T+d d l+E F Z$ & 4 & 1,1 & $6,8 \pm 1,6$ & 6,9 & 24,2 \\
\hline$A Z T+3 T C$ & 4 & 1,1 & $6,9 \pm 1,4$ & 6,6 & 20,2 \\
\hline$A Z T+d d l$ & 1 & 0,3 & $6,4 \pm 0,0$ & - & 0,0 \\
\hline$d d l+d 4 T+N V P$ & 1 & 0,3 & $7,8 \pm 0,0$ & - & 0,0 \\
\hline $3 T C+d 4 T+I D V$ & 1 & 0,3 & $2,4 \pm 0,0$ & - & 0,0 \\
\hline $3 T C+d 4 T+I D V+R T V$ & 1 & 0,3 & $8,7 \pm 0,0$ & - & 0,0 \\
\hline$A Z T+3 T C+R T V+S Q V$ & 1 & 0,3 & $6,3 \pm 0,0$ & - & 0,0 \\
\hline $3 T C+d 4 T+L P V+R T V$ & 1 & 0,3 & $4,9 \pm 0,0$ & - & 0,0 \\
\hline$A Z T+3 T C+L P V+R T V$ & 1 & 0,3 & $5,4 \pm 0,0$ & - & 0,0 \\
\hline Global & 358 & 100,0 & $7,7 \pm 1,5$ & 8,1 & 19,3 \\
\hline
\end{tabular}

ITRN = Inibidores da transcriptase reversa análogos de nucleosídeo: $A Z T=$ Zidovudina; ddl = Didanosina;

$\mathrm{d} 4 \mathrm{~T}=$ Estavudina; $3 T \mathrm{C}=$ Lamivudina. ITRNN = Inibidores da transcriptase reversa não-análogos de nucleosídeo:

NVP = Nevirapina; EFZ = Efavirenz. IP = Inibidores da Protease: NFV = Nelfinavir; IDV = Indinavir; RTV = Ritonavir; $\mathrm{SQV}=$ Saquinavir; LPV = Lopinavir. DP = Desvio padrão; CV = Coeficiente de variação. 
do esquema triplo mostrou-se mais elevada do que em trabalhos anteriormente desenvolvidos, como nos estudos de Lignani Jr. et al. 16 e Bomtempo 17, indicando mudanças de conduta de acordo com os consensos nacionais.

A orientação ao paciente sobre seus medicamentos e o modo correto de usá-los, deveria se constituir em um aspecto prioritário e intrínseco à terapêutica medicamentosa, envolvendo a participação de todos os profissionais de saúde que o assistem. Este estudo constatou que a maioria dos participantes foi orientada por dois profissionais de saúde, situação que oferece maior oportunidade de esclarecimento de eventuais dúvidas por parte dos pacientes. Para aqueles pacientes que não receberam a orientação do profissional de saúde, é possível que uma parcela seja atendida em rede particular e apenas busquem os medicamentos na rede pública, distribuídos gratuitamente. $\mathrm{O}$ conhecimento sobre o modo de ação dos anti-retrovirais, conteúdo que a atividade de orientação deveria contemplar, foi aferido por meio de perguntas feitas durante a entrevista basal, antes de se proceder a comparação das respostas do indivíduo com a prescrição. Verificou-se que em torno de um quarto da população investigada não sabia ou tinha informações equivocadas a respeito da terapia. Além disso, cerca de $20,0 \%$ desconheciam a necessidade de se tomar os medicamentos durante toda a vida. Resultados semelhantes foram achados em outros estudos sobre doenças crônicas, como em Boulet 10, o qual relata grande proporção de pacientes que não compreenderam o papel dos medicamentos e tinham várias idéias equivocadas, reduzindo então a disposição para usá-los.

Para os pacientes com HIV/AIDS, o processo de tratamento é doloroso, repleto de dificuldades a serem superadas, como o momento do conhecimento da infecção pelo HIV ou do reconhecimento da evolução da AIDS implícito no início do tratamento, a estigmatização da doença, a adaptação da rotina da terapia no seu estilo de vida, além de inúmeras reações adversas. $\mathrm{O}$ início do tratamento mostra com mais transparência a necessidade de aceitação da doença e do estabelecimento de relação confiável com o médico e o serviço de saúde 3 . Diversos estudos constataram que uma taxa de adesão precoce é um fator preditivo de uma boa adesão em longo prazo 5 . Essa constatação reforça a importância da atividade de orientação no início do tratamento. Nessa perspectiva, o presente estudo investigou pacientes que estavam sendo admitidos para a primeira prescrição anti-retroviral e enfocou as orientações realizadas pelo médico e farmacêutico relativas a esses medicamentos, bem como o nível de entendimento dessas orientações, na percepção dos pacientes. Foram observadas lacunas relevantes quanto à orientação de aspectos importantes relacionados à terapia ARV e no entendimento dessas orientações. O estudo constatou ainda que houve uma maior proporção de pacientes com orientação farmacêutica, como também uma maior proporção de pacientes que entendeu completamente a orientação farmacêutica, quando comparamos com os resultados da orientação médica. Esse resultado está em conformidade com vários estudos que observaram falhas de comunicação na relação entre médicos e pacientes 6,8,18. O estudo detalhado do processo de comunicação entre médicos ou outros profissionais de saúde e os pacientes é essencial para melhorar o cuidado médico 19. Uma possível explicação para o ocorrido é o fato de que os pacientes passam pela orientação farmacêutica após terem sido consultados pelo médico. O farmacêutico reforça as instruções fornecidas pelos médicos e, além disso, dispensa um tempo maior para os temas específicos da terapia prescrita. A atuação do farmacêutico visando a promover a melhoria da qualidade das informações sobre o esquema terapêutico tem sido apontada em vários estudos que abordam esse tema, como por exemplo, o desenvolvido por Kessler 8, mostrando que $64,0 \%$ a $89,0 \%$ dos entrevistados queriam ser informados pelos seus farmacêuticos sobre como tomar os medicamentos, como estocar, quais os possíveis efeitos colaterais, e se algum tipo de alimentação ou outros medicamentos deveriam ser evitados durante o uso da medicação.

Este estudo comparou o porcentual de concordância entre as informações registradas na receita médica e as fornecidas pelo paciente, para todos os medicamentos anti-retrovirais prescritos. Dentre os itens considerados de grande importância para o uso seguro dos medicamentos - nome, dose e freqüência - observamos a menor proporção de concordância para o item nome. A proporção dessa divergência para o nome do medicamento $(27,5 \%)$ foi cerca de três vezes maior que o índice de divergência para a freqüência $(8,3 \%)$ e para a dose $(7,2 \%)$. Esse resultado pode ser devido à complexidade e diversidade dos nomes dos anti-retrovirais, o que dificulta a memorização, aliada à baixa escolaridade de grande parte dos entrevistados. Ressalte-se que as respostas dadas em nome comercial ou em siglas para medicamento prescrito utilizando-se o nome do fármaco e vice-versa eram aceitas. É interessante mencionar, ainda, que foi permitido ao paciente con- 
sultar a receita ou a embalagem do medicamento, ou qualquer anotação que ele tivesse em mãos. Considerando-se a complexidade que permeia a terapia anti-retroviral e que tais informações são imprescindíveis ao adequado cumprimento da prescrição médica, os resultados encontrados mostram-se potencialmente críticos, podendo influenciar a não-adesão ao tratamento. Resultados semelhantes foram encontrados por Silva et al. 15, Olivares \& Espinoza 20 e Tempier 21 . Um outro trabalho enfocando o impacto da comunicação entre médico e paciente, portador de doença crônica, encontrou que pacientes que aprenderam os nomes de sua medicação foram mais aderentes do que aqueles que não sabiam os nomes 22 . Em relação a informações relativas aos itens reações adversas, precauções a serem adotadas e recomendações quanto à alimentação, observou-se no presente estudo uma proporção muito baixa de registro nas receitas médicas. A falta de informações relativas a reações adversas nas receitas médicas aqui encontradas está de acordo com o trabalho de Silva et al. 15 e Tempier 21, que sublinham a probabilidade dessa informação ser omitida muitas vezes pelo prescritor e pelo farmacêutico, temerosos de influenciar o tratamento do paciente e interferir na adesão, caso o paciente por auto-sugestão venha a sentir os efeitos alertados pelo profissional de saúde.

Apesar do predomínio da resposta afirmativa dos entrevistados quanto a terem sido orientados sobre sua alimentação a cada ingestão de anti-retroviral, informações sobre este item foram registradas para apenas $13,2 \%$ dos antiretrovirais nas receitas. O índice de concordância relativo à alimentação mostrou que os pacientes desconheciam ou estavam equivocados em relação a essa orientação para 52,3\% dos anti-retrovirais. Este fato é preocupante, uma vez que recomendações sobre a interação dos anti-retrovirais com alimento estão presentes no Consenso Brasileiro 5, sendo algumas específicas para determinados medicamentos e importantes para seu uso correto e absorção adequada. Para exemplificar, o DDI só deve ser administrado $\geq 30$ minutos antes ou $\geq 2$ horas após a alimentação e o Nelfinavir deve ser administrado com alimentos. Também se observou uma proporção muito baixa de registro referente às precauções e em apenas $15,3 \%$ dos anti-retrovirais as respostas dos pacientes foram concordantes com a prescrição/consenso. Ao mesmo tempo, 15,1\% dos pacientes entrevistados desconheciam a duração do tratamento e $28,8 \%$ deles erraram informações relativas à indicação terapêutica. Assim, é expressiva a porcentagem de pacientes portadores de HIV/AIDS que desconhecem os itens descritos acima, fato que pode comprometer o grau de adesão desejado, em torno de $90 \%$ a $100 \%$. As proporções de concordância para esses itens, encontrados em nosso estudo, são próximas daquelas encontradas por Hulka et al. 22, Tempier 21, Silva et al. 15 e Stone et al. 23. Metha et al. 4 encontraram relação entre melhora dos sintomas e interrupção da medicação entre um grupo de não-aderentes, o que mostra a importância de explicar a esses pacientes a necessidade de manter o uso da medicação durante o período assintomático.

$\mathrm{O}$ esquema mais prescrito foi AZT + 3TC associado ao Nelfinavir [2 ITRN + 1 IP], seguido pelo AZT + 3TC associado ao Efavirenz [2 ITRN +1 ITRNN] e AZT + 3TC associado ao Nevirapina [2 ITRN + 1 ITRNN]. De acordo com o Consenso Brasileiro 5, na terapia inicial, os esquemas preferenciais recomendados variam de acordo com o estágio da infecção pelo HIV. Neste estudo, aproximadamente metade dos pacientes iniciou o tratamento já com o esquema incluindo o inibidor de protease. $\mathrm{O}$ atual Consenso Brasileiro 5, ao enfocar as opções entre esquemas triplos com que deve ser iniciada a terapia, passou a recomendar preferencialmente um ITRNN como a terceira droga a ser associada a dois ITRN, independentemente da contagem de linfócitos T-CD4+, considerando os esquemas com inibidores da protease como alternativa para o início de terapia anti-retroviral em pacientes virgens de tratamento. A escolha dos medicamentos por grupo farmacológico mostrou concordância com o Consenso Brasileiro ${ }^{5}$. A utilização de outra medicação de uso contínuo ou regular, diferente dos anti-retrovirais ocorreu para 40,5\% dos participantes, proporção menor do que a encontrada $(66,5 \%)$ por Bomtempo 17. Uma explicação plausível seria que a menor proporção de profiláticos, observada no presente trabalho, pode estar relacionada a um maior uso de esquema tríplice e de inibidores de protease, apresentando resultados mais eficazes no controle da evolução da doença.

Considerando a mediana, os melhores níveis de compreensão foram observados para os esquemas AZT + ddI + Nelfinavir e AZT + ddI + Indinavir. Esses medicamentos apresentam uma diversidade quanto à apresentação (comprimidos, cápsulas, comprimidos tamponados para diluir em água ou mastigar), posologia, restrição alimentar, precauções que ainda variam intrinsecamente com as associações, facilitando ou dificultando o seu uso. Esses fatores podem dificultar inferências que justifiquem as diferenças 
do nível de compreensão observadas entre os esquemas prescritos. Assim, por exemplo, o ddI, presente nos dois esquemas com melhores níveis de compreensão, comprimido tamponado, necessita ser administrado $\geq 30$ minutos antes ou $\geq 2$ horas após alimentação, devendo ser diluído em água ou mastigado. A associação AZT + ddI com maior número de comprimidos, por isso mais complexa, é sugerida como opção terapêutica de segunda escolha. O ddI diminui a absorção do Indinavir e Nelfinavir, por isso recomenda-se administrar com intervalo mínimo de uma hora 5 . Em síntese, toda essa circunstância, aumenta a complexidade do tratamento, o que torna intrigante o resultado obtido. Uma provável explicação seria a exigência de um esforço maior por parte dos profissionais de saúde no ato de promover as orientações, diante da complexidade dos esquemas citados.

No presente estudo, o cálculo do nível global de compreensão da prescrição de anti-retroviral foi possível para 358 entrevistados, apresentou média de 7,7 (DP = 1,5; CV = 19,3\%) e mediana de 8,1 , tendo sido classificado como insuficiente $(<7,0)$ para $94(26,3 \%)$. Esse resultado preocupa e indica a necessidade de se aprofundar a análise, visando a identificar os fatores que se relacionam com o nível insuficiente de compreensão da terapia anti-retroviral.

É expressiva a porcentagem de pacientes com nível insuficiente de compreensão, o que pode significar um risco em potencial para o não cumprimento da prescrição. Os resultados obtidos revelaram inadequações nas orienta- ções dadas pelos profissionais da saúde e pacientes com lacunas importantes na compreensão de informações sobre a terapêutica anti-retroviral. Portanto, é necessário investir em estratégias que incrementem o número e a qualidade das orientações fornecidas a esses pacientes, pois um nível insuficiente de compreensão pode contribuir para uma baixa adesão dos pacientes em tratamento. É fundamental o papel da comunicação profissional de saúde-paciente na implementação dessas estratégias. A adoção de um enfoque multidisciplinar no atendimento aos pacientes portadores do HIV pode ajudar a reverter a situação observada. A participação do farmacêutico no trabalho em equipe mostra-se essencial, especialmente quando existe uma maior complexidade terapêutica e, portanto, uma maior dificuldade na compreensão pelo paciente quanto ao seu tratamento. No esforço de ampliar essa compreensão também deverão ser estimuladas as intervenções múltiplas, combinando estratégias educacionais e comportamentais, as abordagens individualizadas principalmente daqueles pacientes considerados de alto risco, além de um adequado monitoramento do tratamento preconizado. Concluímos que vários aspectos identificados por este estudo como dificultadores da compreensão do tratamento anti-retroviral são passíveis de modificação e, nesse sentido, seu enfrentamento poderia contribuir para melhorar a adesão a esse tratamento e resultar em maior efetividade na assistência aos portadores do HIV/AIDS e de outras doenças crônicas.

\section{Resumo}

Para avaliar a compreensão das informações sobre a terapia anti-retroviral entre portadores do HIVIAIDS atendidos em serviços públicos de referência, em Belo Horizonte, Minas Gerais, Brasil, foi realizada análise transversal por meio de entrevistas com pacientes, após a primeira dispensação de anti-retrovirais. A orientação recebida dos profissionais de saúde e o nível de compreensão pelo paciente foram investigados, sendo este classificado como insuficiente se houvesse discordância igual ou superior a 30,0\% entre a resposta do paciente e a informação contida na prescrição para itens selecionados. Divergências entre as informações da prescrição e as relatadas pelos 358 pacien- tes foram observadas. O nível de compreensão das informações sobre anti-retrovirais prescritos foi classificado como insuficiente para 26,3\%. Os resultados obtidos revelam proporção importante de pacientes com lacunas na compreensão de informações sobre a terapêutica anti-retroviral combinada. É necessário implementar estratégias para aumentar a qualidade das orientações fornecidas a esses pacientes. O enfoque multidisciplinar no atendimento aos pacientes poderá contribuir para reverter a situação observada.

Síndrome de Imunodeficiência Adquirida; Terapia Anti-Retroviral de Alta Atividade; Análise Transversal 


\section{Colaboradores}

M. G. B. Ceccato, F. A. Acurcio, P. F. Bonolo e M. D. C. Guimarães contribuíram no desenho do estudo, análise e interpretação de dados, elaboração e revisão crítica do texto e aprovação da versão final do artigo. G. M. Rocha participou da análise e interpretação de dados.

\section{Agradecimentos}

A realização deste trabalho só foi possível com o apoio financeiro da Organização Pan-Americana da Saúde/World Health Organization e Programa Nacional de DST e AIDS (PN-DST e AIDS) do Ministério da Saúde, além do apoio logístico da Coordenação Estadual de DST e AIDS (CE-DST e AIDS) da Secretaria Estadual de Saúde de Minas Gerais, Núcleo de Estudos em Saúde Coletiva (NESCON), Grupo de Pesquisas em Epidemiologia e Avaliação em Saúde (GPEAS), Departamentos de Medicina Preventiva e Social e de Farmácia Social, da Universidade Federal de Minas Gerais.

14. Isacson D, Bingefors K. Attitudes towards drugs a survey in the general populations. Pharm World Sci 2002; 24:104-10.

15. Silva T, Schenkel EP, Mengue SS. Nível de informação a respeito de medicamentos prescritos a pacientes ambulatoriais de hospital universitário. Cad Saúde Pública 2000; 16:449-55.

16. Lignani Jr. LL, Greco DB, Carneiro M. Avaliação da aderência aos anti-retrovirais em pacientes com infecção pelo HIV/Aids. Rev Saúde Pública 2001; 35:495-501.

17. Bomtempo NM. Estudo de fatores de risco para uso irregular do tratamento anti-retroviral, em um serviço público de referência, em Minas Gerais, 1998-1999 [Dissertação de Mestrado]. Belo Horizonte: Faculdade de Medicina, Universidade Federal de Minas Gerais; 2000.

18. Bakirtzief $Z$. Identificando barreiras para adesão ao tratamento de hanseníase. Cad Saúde Pública 1996; 12:497-505.

19. Korsch BM, Negrete VF. Doctor-patient communication. Sci Am 1972; 227:66-74.

20. Olivares J, Espinoza Y. Determinación del nível de información sobre el uso de antiinflamatorios no esteroideos en pacientes ambulatorios de un hospital general. Medicamentos y Salud Popular 1996; 33:19-28.

21. Tempier R. Long-term psychiatric patient's knowledge about their medication. Psychiatr Serv 1996; 47:1385-7.

22. Hulka BS, Cassel JC, Kupper LL, Burdette JA. Communication, compliance, and concordance between physicians and patients with a prescribed medications. Am J Public Health 1976; 66:847-53.

23. Stone VE, Hogan JW, Schuman P, Rompalo AM, Howard AA, Korkontzelou C, et al. Antiretroviral regimen complexity, self-reported adherence, and HIV patients' understanding of their regimens: survey of women in the her study. J Acquir Immune Defic Syndr 2001; 28:124-31.

Recebido em 15/Dez/2003

Versão final reapresentada em 18/Mai/2004

Aprovado em 27/Mai/2004 an position paper. Can J Hosp Pharm 2000; 53:92103. 